\title{
Contaminantes de preocupação emergente no Brasil na década 2010- 2019 - Parte I: ocorrência em diversos ambientes aquáticos
}

\author{
Contaminants of emerging concern in Brazil in the decade \\ 2010-2019 - Part I: occurrence in various aquatic \\ environments
}

Cindy Deina Farto ${ }^{1}$ (D), Gilson Barbosa Athayde Júnior ${ }^{1}$ (D), Rennio Félix de Sena ${ }^{1}$ (D), Raul Rosenhaim ${ }^{1}$

1Universidade Federal da Paraíba - UFPB, João Pessoa, PB, Brasil. E-mails: cindy.deina@academico.ufpb.br, gilson@ct.ufpb.br, rennio@ct.ufpb.br, raulrosenhaim@gmail.com

\begin{abstract}
Como citar: Farto, C. D., Athayde Júnior, G. B., Sena, R. F., \& Rosenhaim, R. (2021). Contaminantes de preocupação emergente no Brasil na década 2010-2019 - Parte I: ocorrência em diversos ambientes aquáticos. Revista de Gestão de Água da América Latina, 18, e6. https://doi.org/10.21168/rega.v18e6
\end{abstract}

RESUMO: 0 objetivo do estudo foi realizar uma comparação dos níveis de concentração de contaminantes de preocupação emergente (CPE) em esgotos brutos e tratados, águas superficiais e águas tratadas no Brasil. Trata-se de um artigo de revisão bibliográfica, no qual buscou-se trabalhos científicos publicados entre os anos de 2010 e 2019 , que trataram sobre a presença desses compostos nas matrizes aquáticas. No total foram analisados 32 trabalhos referentes a presença desses compostos, sendo 7 deles em esgotos (bruto e tratado) e 25 em águas (superficiais e tratadas). Os contaminantes de preocupação emergente estão presentes em águas do território brasileiro, podendo ocorrer um decréscimo em suas concentrações ao longo da seguinte sequência de ambientes aquáticos: esgoto bruto, esgoto tratado, águas superficiais (rios) e águas de abastecimento público. As estações de tratamento de esgoto (ETEs) apresentam alguma remoção destes compostos, embora de magnitude reduzida, enquanto há algumas evidências de que o mesmo não ocorre nas estações de tratamento de água (ETAs).

Palavras-chave: Contaminantes de Preocupação Emergente; Ocorrência; ETEs; ETAs; Águas Superficiais.

ABSTRACT: The aim of this study was to discuss the state-to-art in relation to the occurrence of contaminants of emerging concern (CECs) in water collections in Brazil. For this, scientific papers published between 2010 and 2019 , which dealt with the presence of these compounds in raw and treated sewage, as well as in surface waters and treated water, were searched. A total of 32 studies were analysed regarding the presence of these compounds in aquatic matrices, 7 of them in sewers (raw and treated) and 25 in water (surface and treated). CECs are present in Brazilian waters, and there may present a decrease in their concentrations along the following sequence of aquatic environments: raw sewage, treated sewage, surface waters (rivers) and public water supply. Sewage treatment stations seem to provide some removal efficiencies although limited, for these compounds, while there is some evidence that the same does not occur in water treatment stations.

Keywords: Contaminants of Emerging Concern; Occurrence; Effluent Treatment Plants; Water Treatment Plants; Surface Qaters.

\section{INTRODUÇÃO}

Os contaminantes de preocupação emergente (CPEs) compreendem uma ampla classe de substâncias de origem tanto antropogênica como natural, compostas na maior parte por compostos farmacêuticos ativos (ex.: antibióticos, anti-inflamatórios, hormônios, dentre outros), produtos de higiene pessoal, agrotóxicos, drogas ilícitas, subprodutos provenientes de processos de desinfecção de águas, produtos industriais (ex.: retardantes de chama e plastificantes, dentre estes) e microplásticos. Além desses, alguns microrganismos e toxinas produzidas por algas também são considerados

Recebido: Fevereiro 23, 2021. Revisado: Abril 21, 2021. Aceito: Maio 19, 2021. 
contaminantes de preocupação emergente (Richardson \& Ternes, 2011; Luo et al., 2014; Queiroz et al., 2014; Richardson \& Kimura, 2016; Montagner et al., 2017; Montagner et al., 2019).

Os CPEs estão relacionados a contaminantes não legislados, ou seja, que não estão incluídos nos programas de monitoramento de rotina, mas que podem ser candidatos a regulamentação futura dependendo da pesquisa sobre os efeitos potenciais para a saúde e os resultados do monitoramento da sua ocorrência (Montagner et al., 2017; Araújo et al., 2019).

Os CPEs só puderam ser detectados e quantificados recentemente por meio do desenvolvimento de novas técnicas analíticas sensíveis e mais complexas, tais como a cromatografia gasosa ou líquida acoplada a espectrometria de massas (GC-MS e LC-MS). Na literatura também se faz uso das seguintes terminologias para CPEs: contaminantes emergentes, poluentes emergentes, micropoluentes, microcontaminantes e microcontaminantes de interesse emergente. (Bila \& Dezzotti, 2007). Neste contexto, o prefixo "micro" é utilizado em referência às baixas concentrações nas quais os CPEs são geralmente encontrados em meio aquático, que é da ordem de $\mu \mathrm{g} \mathrm{L}^{-1}$, podendo variar, no entanto, tanto para mais como para menos.

Alguns CPEs são considerados como disruptores endócrinos (DEs), que são compostos que mimetizam ou bloqueiam a ação dos hormônios naturais. Esses compostos exógenos ligam-se aos receptores celulares alterando suas respostas normais. Os DEs podem intervir no sistema endócrino de humanos e de outros animais, podendo causar declínio da qualidade do sêmen, infertilidade, diabetes, hipo/hipertireoidismo, aumento da incidência de câncer de mama, de testículo e de próstata e a endometriose (Bila \& Dezzotti, 2007; Crain et al., 2008; Sodré, 2012; Aquino et al., 2013; Alves et al., 2017).

De acordo com Bila \& Dezzotti (2007), os DEs podem ser tanto substâncias naturais quanto sintéticas, dentre os quais podem ser citados: pesticidas como atrazina; subprodutos da degradação de alquilfenóis polietoxilados como 4-nonilfenol; hidrocarbonetos poliaromáticos; aditivos plastificantes como dietil-hexilftalato; compostos industriais como bisfenol A; metais pesados como cádmio, mercúrio e chumbo; hormônios sintéticos como dietilestilbestrol e $17 \alpha$-etinilestradiol; fitoestrogênios como genisteína e metaresinol e os hormônios naturais estrona, $17 \beta$-estradiol e estriol.

Ocorrências de CPEs em ambientes aquáticos têm sido observadas nas últimas décadas, tais como as relatadas por Stumpf et al. (1999), Ribeiro et al. (2009), Jelic et al. (2011), Montagner \& Jardim (2011), Santos et al. (2013), Rattier et al. (2014) e Fairbairn et al. (2016), Sousa et al. (2018), Sposito et al. (2018) e Pivetta \& Gastaldini (2019), sendo capazes de causar prejuízos tanto à saúde humana, quanto ambiental.

De acordo com Raimundo (2011), o destino e o efeito de CPEs em águas brasileiras ainda têm sido pouco discutidos na literatura. Contudo, tem-se que a principal via de aporte deles em águas superficiais não está relacionada à contribuição dos efluentes de ETE, mas sim ao aporte de esgoto bruto. A Figura 1 apresenta a porcentagem de esgotos sanitários tratados no Brasil entre os anos de 2010 e 2018. Observa-se que o percentual de esgotos sanitários tratados no Brasil ainda é baixo, uma vez que no ano de 2018 , somente $46 \%$ do esgoto sanitário gerado no país, foram tratados. Na região Norte e Nordeste, os números são mais preocupantes, pois, no ano de 2019, apenas 22\% e 33,7\%, respectivamente, do esgoto gerado foi tratado (Sistema Nacional de Informações sobre Saneamento, 2020). Dessa maneira, diversos municípios descartam o esgoto bruto diretamente nos rios, mesmo aqueles que são utilizados como fonte de água para abastecimento público da região.

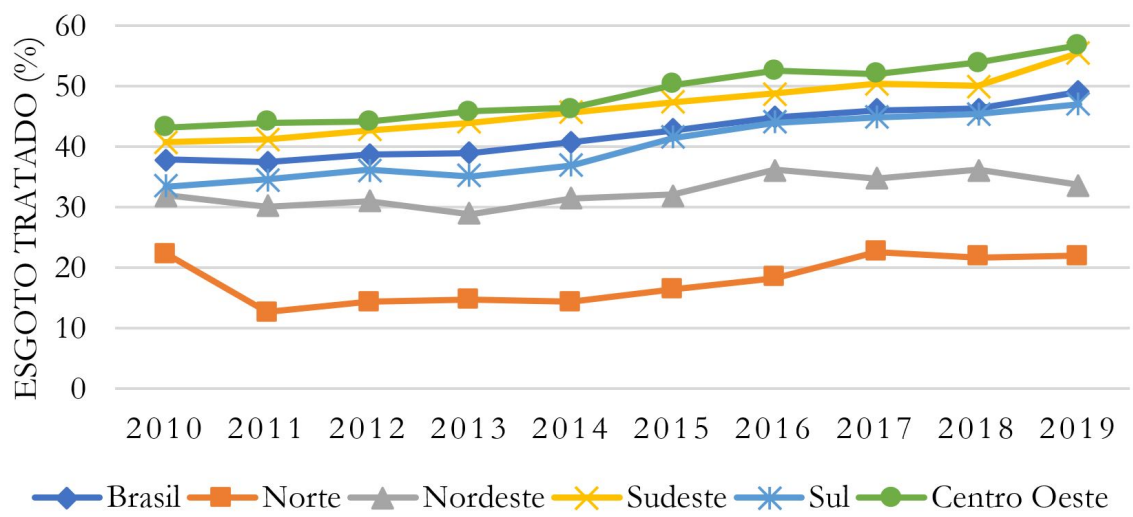

Figura 1. Percentual de esgotos tratados segundo macrorregião geográfica e Brasil nos anos de 2010 a 2019 Fonte. Sistema Nacional de Informações sobre Saneamento (2020)

A preocupação com relação à presença dos CPEs em águas brasileiras surge em função de dois aspectos distintos: (a) a exposição da biota e dos seres humanos a esses compostos, seja via recreacional ou por meio da ingestão e/ou absorção dérmica e (b) a possibilidade de contaminação de 
mananciais de água bruta, com transferência destas substâncias para as ETAs e para a água destinada ao abastecimento público (Raimundo, 2011). Como muitos CPEs são compostos de difícil degradação, o problema torna-se maior, pois os tratamentos convencionais (para água de abastecimento) e biológicos (para esgotos) não removem de forma satisfatória esses contaminantes da água, podendo afetar a qualidade da água potável produzida (Alves et al., 2017).

Outro fator preocupante em relação aos CPEs em coleções hídricas está relacionado às práticas agrícolas intensivas que levam à contaminação do solo e consequentemente podem causar a lixiviação de poluentes para as águas superficiais e subterrâneas, chegando até mesmo à água tratada (Gavrilescu et al., 2015). Ainda no meio rural, os fármacos de uso veterinário contribuem ativamente na disposição de antibióticos empregados no tratamento terapêutico dos animais e de hormônios administrados para acelerar o crescimento ou regular o sistema reprodutivo. 0 escoamento superficial e o vazamento de fossas sépticas e aterros sanitários também atuam como fontes de dispersão desses contaminantes orgânicos (Fairbairn et al., 2016).

Diante do que foi exposto, o presente trabalho tem como objetivo realizar uma comparação dos níveis de concentração de contaminantes de preocupação emergente em esgotos brutos e tratados, águas superficiais e águas tratadas no Brasil.

\section{METODOLOGIA}

Esta revisão foi realizada mediante busca eletrônica de artigos que tratavam sobre a ocorrência de CPEs no Brasil, indexados nas seguintes bases de dados: Web of Science, Science Direct, SciELO e Scopus. Tais bases de dados científicos foram escolhidas conforme relevância e expressão no meio científico, com amplo acervo na área de engenharias. As buscas foram realizadas a partir dos seguintes descritores: "Contaminantes de preocupação emergente", "Contaminantes emergentes", "Disruptores endócrinos", "Ocorrência”. "Esgoto”, "Água superficial”, "Água tratada”. Esses descritores foram interligados pelos operadores booleanos "AND" e "OR" para favorecer a busca dos estudos pelas bases de dados.

Foram incluídos textos completos disponíveis eletronicamente, no idioma português, inglês e espanhol, no período de janeiro de 2010 a dezembro de 2019, e que apresentavam a temática proposta no título, no resumo ou nos descritores. Quando considerado pertinente, outras bibliografias foram consultadas a partir de referências dos artigos previamente analisados. Sendo assim, neste trabalho de revisão foram considerados algumas dissertações e teses, bem como trabalhos publicados em revistas não indexadas, a fim de se obter uma maior quantidade de dados para representar mais seguramente o cenário brasileiro no tocante aos CPEs.

Os critérios de exclusão foram: trabalhos que não foram publicados no período considerado, além dos artigos que não realizaram algum tipo de análise nesse âmbito, ou seja, não avaliaram ocorrência de CPEs em coleções hídricas no Brasil.

A Figura 2 apresenta a evolução temporal dos estudos publicados de acordo com a matriz aquática estudada. No total foram analisados 32 estudos referentes a presença dos CPEs, sendo 7 deles em esgoto bruto, 3 em esgoto tratado, 20 em águas superficiais e 8 em águas tratadas. Ressalta-se que 3 estudos se repetiram nas matrizes esgoto bruto e tratado e 3 estudos se repetiram nas matrizes água superficial e água tratada.

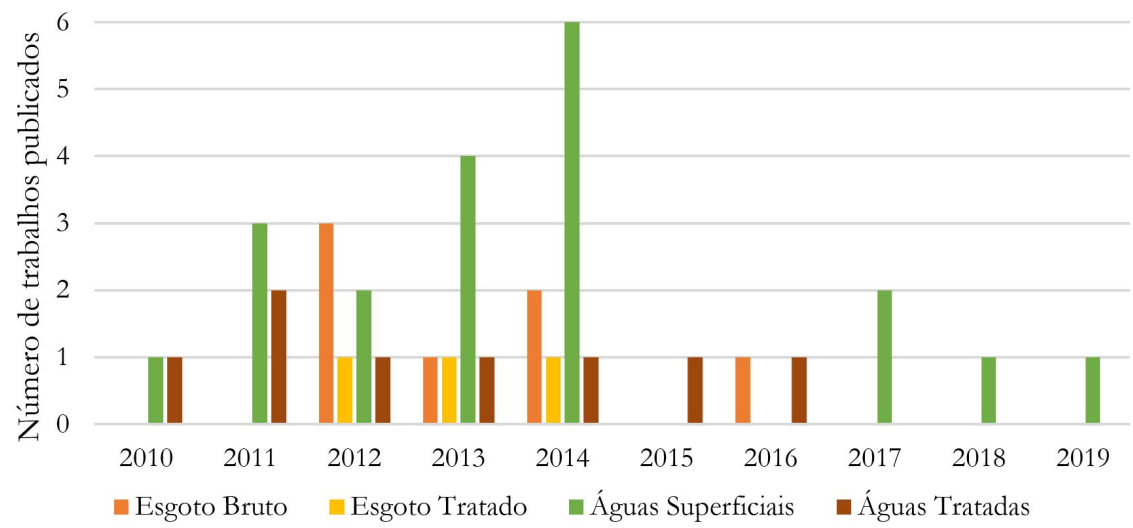

Figura 2. Evolução temporal dos trabalhos publicados de acordo com a matriz aquática 
Os resultados sobre a ocorrência de CPEs foram apresentados sob a forma de quadros. As variáveis para elaboração dos resultados foram: composto, classe, concentração máxima, mínima e média, localidade/estado e referências. Estes itens foram considerados suficientes para descrever os resultados de maneira sucinta e relevante ao objeto de estudo.

\section{OCORRÊNCIAS DE CONTAMINANTES DE PREOCUPAÇÃO EMERGENTE EM COLEÇÕES HÍDRICAS NO BRASIL}

Nos Quadros 1, 2 e 3 encontram-se, respectivamente, uma listagem de estudos acerca da ocorrência de CPEs em ETEs (esgoto bruto e tratado), águas superficiais (rios) e ETAs no Brasil. Em uma visão geográfica sobre o cenário dos CPEs no Brasil, foram encontrados dados de ocorrência de diferentes classes de compostos em diferentes matrizes ambientais em 15 dos 26 estados brasileiros e no Distrito Federal.

\section{ETES}

Sobre a ocorrência dos CPEs em estações de tratamento de esgoto do Brasil, foram encontrados 7 trabalhos que avaliaram a presença e/ou a remoção de 16 compostos em quatro estados (Rio de Janeiro, São Paulo, Minas Gerais e Ceará) e no Distrito Federal.

Dentre os 16 compostos estudados nesta matriz, 8 estão na classe dos fármacos, 4 compostos na classe dos hormônios, 2 compostos são drogas ilícitas, 1 plastificante e 1 produto de uso industrial.

As concentrações dos contaminantes no esgoto bruto variaram quatro ordens de grandeza, entre 0,56 e 63.840,00 ng L-1, refletindo o padrão de consumo de cada região. No esgoto tratado as concentrações variaram entre 0,09 e 13.860,00 ng L ${ }^{-1}$. A presença destas substâncias nos diversos efluentes é preocupante, pois eles podem ser considerados a maior fonte de contaminação para o ambiente.

Quadro 1. Concentrações de contaminantes de preocupação emergente determinados em ETEs no Brasil

\begin{tabular}{|c|c|c|c|c|c|}
\hline Composto & Classe & $\begin{array}{l}\text { Concentração } \\
\text { máxima - } \\
\text { esgoto bruto } \\
\left.\text { (ng } L^{-1}\right)\end{array}$ & $\begin{array}{c}\text { Concentração } \\
\text { máxima- } \\
\text { esgoto tratado } \\
\left(n g L^{-1}\right)\end{array}$ & Localidade/Estado & Referência \\
\hline \multirow{4}{*}{17 - $\beta$-estradiol } & \multirow{4}{*}{ Hormônio } & 776,00 & 397,00 & $-/ \mathrm{CE}$ & $\begin{array}{c}\text { Pessoa et al. } \\
\text { (2014) }\end{array}$ \\
\hline & & 1,24 & 0,34 & $\begin{array}{c}\text { Ilha do } \\
\text { Governador/RJ }\end{array}$ & \multirow{2}{*}{$\begin{array}{c}\text { Ferreira } \\
(2013)\end{array}$} \\
\hline & & 1,11 & 0,23 & Penha/RJ & \\
\hline & & $2.520,00$ & $1.240,00$ & Fortaleza/CE & $\begin{array}{c}\text { De Paiva } \\
\text { Pessoa et al. } \\
(2012)\end{array}$ \\
\hline \multirow{4}{*}{ Bisfenol A } & \multirow{4}{*}{ Plastificante } & 165,00 & - & Belo Horizonte/MG & $\begin{array}{c}\text { Queiroz et al. } \\
(2014)\end{array}$ \\
\hline & & $2.770,00$ & 670,00 & $\begin{array}{c}\text { Ilha do } \\
\text { Governador/RJ }\end{array}$ & \multirow{2}{*}{$\begin{array}{c}\text { Ferreira } \\
\text { (2013) }\end{array}$} \\
\hline & & $5.310,00$ & $1.790,00$ & Penha/RJ & \\
\hline & & 308,80 & - & Belo Horizonte/MG & $\begin{array}{c}\text { Queiroz et al. } \\
\text { (2012) }\end{array}$ \\
\hline \multirow{4}{*}{ Diclofenaco } & \multirow{4}{*}{ Fármaco } & $2.380,00$ & - & São Carlos/SP & \multirow{2}{*}{$\begin{array}{c}\text { Grosseli } \\
(2016)\end{array}$} \\
\hline & & $3.352,00$ & - & Jundiaí/SP & \\
\hline & & 100,00 & - & \multirow{2}{*}{ Belo Horizonte/MG } & $\begin{array}{l}\text { Queiroz et al. } \\
\text { (2014) }\end{array}$ \\
\hline & & 240,20 & - & & $\begin{array}{c}\text { Queiroz et al. } \\
\text { (2012) }\end{array}$ \\
\hline \multirow[b]{2}{*}{ Estrona } & \multirow[b]{2}{*}{ Hormônio } & $3.050,00$ & $2.080,00$ & $\mathrm{CE}$ & $\begin{array}{c}\text { Pessoa et al. } \\
\text { (2014) }\end{array}$ \\
\hline & & $2.570,00$ & $2.080,00$ & Fortaleza/CE & $\begin{array}{c}\text { De Paiva } \\
\text { Pessoa et al. } \\
(2012)\end{array}$ \\
\hline
\end{tabular}




\begin{tabular}{|c|c|c|c|c|c|}
\hline Composto & Classe & $\begin{array}{l}\text { Concentração } \\
\text { máxima - } \\
\text { esgoto bruto } \\
\left.\text { (ng } L^{-1}\right)\end{array}$ & $\begin{array}{l}\text { Concentração } \\
\text { máxima- } \\
\text { esgoto tratado } \\
\left(n g L^{-1}\right)\end{array}$ & Localidade/Estado & Referência \\
\hline \multirow{2}{*}{ Sulfametoxazol } & \multirow{14}{*}{ Fármaco } & 13,00 & - & \multirow{4}{*}{ Belo Horizonte/MG } & $\begin{array}{c}\text { Queiroz et al. } \\
(2014)\end{array}$ \\
\hline & & 150,80 & - & & $\begin{array}{l}\text { Queiroz et al. } \\
\qquad(2012)\end{array}$ \\
\hline \multirow{2}{*}{ Trimetoprim } & & 61,00 & - & & $\begin{array}{l}\text { Queiroz et al. } \\
\text { (2014) }\end{array}$ \\
\hline & & 113,70 & - & & $\begin{array}{l}\text { Queiroz et al. } \\
\text { (2012) }\end{array}$ \\
\hline \multirow{2}{*}{ Atenolol } & & $51.036,00$ & - & São Carlos/SP & \multirow{10}{*}{$\begin{array}{l}\text { Grosseli } \\
\text { (2016) }\end{array}$} \\
\hline & & $37.619,00$ & - & Jundiaí/SP & \\
\hline \multirow{2}{*}{ Carbamazepina } & & 853,00 & - & São Carlos/SP & \\
\hline & & $2.461,00$ & - & Jundiaí/SP & \\
\hline \multirow{2}{*}{ Ibuprofeno } & & $1.209,00$ & - & São Carlos/SP & \\
\hline & & $3.062,00$ & - & Jundiaí/SP & \\
\hline \multirow{2}{*}{ Naproxeno } & & $1.589,00$ & - & São Carlos/SP & \\
\hline & & $2.901,00$ & - & Jundiaí/SP & \\
\hline \multirow{2}{*}{ Triclosan } & & 678,10 & - & São Carlos/SP & \\
\hline & & 602,70 & - & Jundiaí/SP & \\
\hline \multirow[t]{2}{*}{ Alquilfenóis } & \multirow{2}{*}{$\begin{array}{l}\text { Composto } \\
\text { industrial }\end{array}$} & $47.230,00$ & $7.910,00$ & $\begin{array}{c}\text { Ilha do } \\
\text { Governador/RJ }\end{array}$ & \multirow{2}{*}{$\begin{array}{c}\text { Ferreira } \\
\text { (2013) }\end{array}$} \\
\hline & & $63.840,00$ & $13.860,00$ & Penha/RJ & \\
\hline Cocaína & \multirow{2}{*}{ Droga ilícita } & $3.690,00$ & - & \multirow{2}{*}{ Brasília/DF } & \multirow{2}{*}{$\begin{array}{l}\text { Maldaner et al. } \\
(2012)\end{array}$} \\
\hline Benzoilecgonina & & $9.719,00$ & - & & \\
\hline $\begin{array}{c}17-\alpha- \\
\text { etinilestradiol }\end{array}$ & \multirow{2}{*}{ Hormônio } & $3.180,00$ & 176,00 & $\mathrm{CE}$ & \multirow{2}{*}{$\begin{array}{l}\text { Pessoa et al. } \\
\text { (2014) }\end{array}$} \\
\hline $\begin{array}{c}17-\beta \text {-estradiol } \\
17 \text {-acetato }\end{array}$ & & $2.300,00$ & $1.250,00$ & $\mathrm{CE}$ & \\
\hline
\end{tabular}

De acordo com Queiroz et al. (2014), o esgoto doméstico é uma matriz muito complexa e difícil de trabalhar do ponto de vista analítico, uma vez que pode conter uma diversidade de compostos orgânicos e inorgânicos, todos com maior concentração quando comparados com os contaminantes de interesse. Devido a isso, alguns desses componentes podem interferir nos resultados referentes a quantificação de contaminantes.

Jelic et al. (2011), relatam que a presença de CPE em efluentes de estações de tratamento de esgotos possuem variações espaciais e temporais significativas, as quais são essencialmente explicadas por vários fatores como a taxa de produção local, práticas e vendas de produtos específicos, metabolismo, consumo per capita de água, tecnologia de tratamento, persistência ambiental e eficiência de remoção no processo de tratamento. Paralelamente, Alves et al. (2017), enfatizam que a produção, uso/consumo local dos produtos que contém CPEs são determinantes na magnitude a qual estes chegam até as ETEs.

Du et al. (2014) verificaram que as concentrações dos CPEs em efluentes foram menores no inverno e maiores no verão, devido, possivelmente, a biodegradação promovida a temperaturas mais elevadas e a diluição elevada durante o período chuvoso. Contudo, o volume de precipitação não necessariamente causa maior diluição dos compostos, inclusive, em alguns casos ela tem sido apontada como fator contribuinte para o aumento das ocorrências; quando o escoamento superficial transporta CPEs, resultando em aumento das concentrações (Petrie et al., 2014; Montagner et al., 2017).

De Paiva Pessoa et al. (2012) relatam que as variações encontradas nas concentrações de estrogênios afluentes a ETEs puderam ser atribuídas tanto à variação do uso de água, que dilui para mais ou menos os estrogênios naturais e sintéticos, quanto ao maior ou menor uso de estrogênios sintéticos, principalmente pelo uso de contraceptivos orais. Esse resultado obtido também pode ser 
atribuído ao período correspondente da coleta, sendo a primeira realizada na estação chuvosa e a segunda, na estação seca, no estado do Ceará.

Todos os fármacos relatados por Grosseli (2016) foram encontrados nas amostras de esgoto bruto da cidade de São Carlos e Jundiaí. Além disso, alguns destes contaminantes se mostraram persistentes nas estações de tratamento. A presença dos contaminantes no esgoto tratado indica que as ETE são fontes pontuais de emissão de contaminantes orgânicos nos rios. Além disso, devido ao caráter recalcitrante da carbamazepina e do diclofenaco nas ETEs estudadas, essas moléculas podem ser investigadas enquanto potenciais indicadores na avaliação de processos adotados em ETE, quanto a sua eficiência na remoção de CPE (Grosseli, 2016).

Com relação às drogas ilícitas, a cocaína e o seu metabólito principal, a benzoilecgonina, foram determinadas em concentrações entre 174,00 e 9.717,00 ng L-1 no esgoto bruto de Brasília no ano de 2012. 0 estudo, aplicado à epidemiologia forense, também revelou uma fonte bastante significativa destes contaminantes para os corpos d'água (Maldaner et al., 2012).

Pessoa et al. (2014), avaliaram a remoção de quatro hormônios presentes em concentrações de micrograma por litro em cinco ETEs no Ceará. Dentre os quatro, o estrona foi o que apresentou maiores concentrações, o que sugere que esse estrogênio é excretado em grandes concentrações por humanos ou outras fontes. Além disso, esse composto foi o que se mostrou mais persistente quando comparado aos demais. De acordo com Zorita et al., (2009), a maior ocorrência desse estrogênio devese principalmente ao fato da estrona ser o principal produto da biodegradação do estradiol, durante os processos de tratamento de esgoto.

Ferreira (2013) mostrou que as concentrações de bisfenol A e dos alquilfenóis no esgoto bruto e tratado esteve em níveis preocupantes quando comparados com os resultados obtidos em outros países, como Estados Unidos e Japão (Lee et al., 2005). Esse resultado reflete a necessidade de pesquisa e implementação de um maior controle nas ETEs, fortalecendo o saneamento básico do país, minimizando assim impactos significativos para o meio ambiente.

Em relação a carbamazepina, diclofenaco, ibuprofeno e naproxeno, os resultados apresentados se assemelham aos trabalhos publicados por Bahlmann et al. (2014), Sari et al. (2014), Pedrouzo et al. (2007) e Petrovic etal. (2006), os quais avaliaram a presença desses compostos em ETEs da Alemanha, Portugal, Turquia, Espanha e Croácia.

De acordo com Pessoa et al. (2014), o monitoramento contínuo desses CPEs é necessário para melhorar os processos de tratamento de águas residuais e garantir a segurança das águas. 0 monitoramento também deve ser expandido para incluir outras matrizes, como o lodo gerado nos sistemas de tratamento.

Uma vez que os CPEs presentes nas águas residuárias alcançam os corpos d'água passíveis de serem utilizados como mananciais de abastecimento, as perspectivas de remoção dos mesmos pelo tratamento são importantes para a manutenção do meio ambiente sadio.

\section{CORPOS HÍDRICOS SUPERFICIAIS (RIOS)}

Sobre a ocorrência dos CPEs em corpos hídricos superficiais do Brasil foram encontrados 20 trabalhos que avaliaram a presença de 34 compostos em nove estados (Rio de Janeiro, São Paulo, Minas Gerais, Paraná, Goiás, Rio Grande do Sul, Mato Grosso, Mato Grosso do Sul e Amazonas).

Dentre os 34 compostos estudados nesta matriz, 18 estão na classe dos fármacos, 7 agrotóxicos, 5 compostos na classe dos hormônios, 2 pertencem a drogas ilícitas, 1 plastificante, e 1 estimulante.

A primeira percepção que surge da análise do Quadro 2 aponta para a evidência da presença em águas superficiais de uma grande gama de compostos com características diferentes, como medicamentos, hormônios naturais, sintéticos, produtos industriais e agrotóxicos.

O Quadro 2 mostra que a cafeína, paracetamol, AAS e atrazina são os micropoluentes encontrados em maiores concentrações, atingindo concentrações máximas médias de 174.557,00 ng L-1, $9.867,00 \mathrm{ng} \mathrm{L}^{-1}, 5.600,00 \mathrm{ng} \mathrm{L}^{-1}$ e 3.281,00 $\mathrm{ng} \mathrm{L}^{-1}$, respectivamente, em águas superficiais. Além desses, os hormônios estrona e $17 \beta$-estradiol e o fármaco ibuprofeno, também foram detectados em grande número de estudos.

A atrazina, que foi o herbicida mais estudado, apesar de ser proibido em alguns países, está entre os mais consumidos no Brasil, de acordo com o Instituto Brasileiro do Meio Ambiente e dos Recursos Naturais Renováveis (IBAMA). É um produto de classe toxicológica III, considerado de toxicidade média para humanos e apresenta potencial de periculosidade ambiental classe III, sendo considerado um produto perigoso (Instituto Brasileiro do Meio Ambiente, 2018; Agência Nacional de Vigilância Sanitária, 2018). 
No Brasil, a atrazina é um dos poucos CPEs que possui legislação para sua concentração no ambiente. Essa legislação, contudo, não veio com o intuito de estabelecer limites para a atrazina como contaminante emergente, mas por ela apresentar efeitos carcinogênicos. A resolução 357/05 do Conselho Nacional do Meio Ambiente (CONAMA) (Brasil, 2005) traz o valor máximo permitido (VMP) para diversas substâncias. Nela, o VMP para atrazina é de 2,00 $\mu \mathrm{g} \mathrm{L}^{-1}$ para corpos de água doce das classes 1,2 e 3.

As concentrações de atrazina variaram entre 0,17 e 10,40 $\mu \mathrm{g} \mathrm{L} \mathrm{L}^{-1}$ em águas superficiais, mostrando que os valores encontrados em alguns estudos se encontraram acima do limite estabelecido.

No estudo realizado por Campanha etal. (2014), nas águas superficiais do rio Mojolinho, localizado em São Carlos-SP, as concentrações dos CPEs foram crescentes desde a nascente até após a confluência do Córrego Água Quente e o lançamento do efluente da ETE (os quais ocorrem no mesmo local), onde ocorreram as maiores concentrações encontradas no rio. Nesse local, ocorre o lançamento de esgoto tratado e não tratado, proveniente do Córrego Água Quente, receptor de esgoto in natura de um dos bairros de São Carlos.

Em trabalhos realizados no estado de São Paulo, foram encontradas altas concentrações de alguns CPEs em águas superficiais. Altas concentrações foram atribuídas à pobre condição do Brasil com relação ao saneamento básico, de forma geral (Montagner \& Jardim, 2011; Raimundo, 2011; Dias, 2014; Sousa et al., 2018).

Thomas et al. (2014) investigaram drogas lícitas e ilícitas selecionadas nos riachos urbanos de Manaus, na Amazônia brasileira, e relataram concentrações de até 5.896,00 e 3.582,00 ng L-1 para cocaína e benzoilecgonina, respectivamente. Os autores verificaram que as concentrações de ambos os contaminantes aumentavam a jusante da região urbana, sugerindo uma contínua entrada ao longo de todo o comprimento do fluxo, provavelmente através insumos brutos de esgoto.

Um ponto que merece destaque é que a maioria dos fármacos listados no Quadro 2 são compostos bioativos que podem ser adquiridos diretamente pela população, à exceção dos antibióticos, sem prescrição médica ou limitação de quantidade. Isso, aliado ao fato de inexistirem políticas efetivas de recolhimento de fármacos vencidos, contribui para o maior descarte ambiental de tais compostos, aumentando, consequentemente, a probabilidade de serem detectados nos recursos hídricos.

Outro ponto que merece ser ressaltado é que os contaminantes provenientes das atividades doméstica e industrial foram estudados especialmente nos rios da região sudeste e sul, que concentram o maior número de indústrias e da população brasileira, o que remete à preocupantes cenários de contaminação. Embora os índices de saneamento básico nessas regiões sejam considerados altos, a quantidade de efluentes bruto lançados diretamente nos recursos hídricos somado a baixa vazão dos rios, compromete os usos múltiplos da água.

De acordo com Montagner \& Jardim (2011), o grau de contaminação dos corpos hídricos reflete o uso e ocupação do solo de cada região, assim, está diretamente relacionado com os níveis de saneamento básico, além de fatores como a densidade populacional e o tipo de economia dos diferentes estados brasileiros.

Quadro 2. Concentrações de contaminantes de preocupação emergente determinados em corpos hídricos superficiais no Brasil

\begin{tabular}{|c|c|c|c|c|}
\hline Composto & Classe & $\begin{array}{l}\text { Concentração } \\
\text { máxima }\left(n g \mathrm{~L}^{-1}\right)\end{array}$ & Localidade/Estado & Referência \\
\hline \multirow{8}{*}{$17 \beta$-estradiol } & \multirow{11}{*}{ Hormônio } & 14,80 & São Carlos/SP & Campanha et al. (2014) \\
\hline & & 72,85 & Nova Lima/MG & \multirow{3}{*}{ Dias (2014) } \\
\hline & & 31,00 & Nova Iguaçu/RJ & \\
\hline & & 85,02 & São Paulo/SP & \\
\hline & & $7.350,00$ & Curitiba/PR & Padilha \& Leitzke (2013) \\
\hline & & 5,69 & Caratinga/MG & Sanson (2012) \\
\hline & & $6.806,00$ & Campinas/SP & Montagner \& Jardim (2011) \\
\hline & & 25,80 & Jaboticabal/SP & Lopes et al. (2010) \\
\hline \multirow{3}{*}{ Estrona } & & 28,70 & São Paulo/SP & Sousa et al. (2018) \\
\hline & & 58,50 & São Carlos/SP & Campanha et al. (2014) \\
\hline & & 3,20 & Rio Doce/MG & Rodrigues et al. (2014) \\
\hline
\end{tabular}




\begin{tabular}{|c|c|c|c|c|}
\hline Composto & Classe & $\begin{array}{l}\text { Concentração } \\
\text { máxima (ng L-1) }\end{array}$ & Localidade/Estado & Referência \\
\hline & & 36,28 & Nova Lima/MG & \multirow{3}{*}{ Dias (2014) } \\
\hline & & 78,05 & Nova Iguaçu/RJ & \\
\hline & & 17,40 & São Paulo/SP & \\
\hline & & $2.420,00$ & Curitiba/PR & Padilha \& Leitzke (2013) \\
\hline & & 8,60 & Goiânia/GO & Fonseca (2013) \\
\hline & & 15,87 & Caratinga/MG & Sanson (2012) \\
\hline & & 600,00 & Jaboticabal/SP & Lopes et al. (2010) \\
\hline \multirow{9}{*}{ Ibuprofeno } & \multirow{23}{*}{ Fármaco } & $2.710,00$ & Santa Maria/RS & Pivetta \& Gastaldini (2019) \\
\hline & & 373,00 & São Paulo/SP & Sousa et al. (2018) \\
\hline & & $1.310,00$ & Curitiba/PR & Goulart (2017) \\
\hline & & $2.396,00$ & São Carlos/SP & Campanha et al. (2014) \\
\hline & & $4.155,50$ & Nova Lima/MG & \multirow{3}{*}{ Dias (2014) } \\
\hline & & 35,43 & Nova Iguaçu/RJ & \\
\hline & & 31,29 & São Paulo/SP & \\
\hline & & 358,20 & Goiânia/GO & Fonseca (2013) \\
\hline & & 70,92 & Caratinga/MG & Sanson (2012) \\
\hline \multirow{7}{*}{ Paracetamol } & & $9.900,00$ & Santa Maria/RS & Pivetta \& Gastaldini (2019) \\
\hline & & $11.280,00$ & Curitiba/PR & Goulart (2017) \\
\hline & & $32.924,00$ & São Carlos/SP & Campanha et al. (2014) \\
\hline & & 292,70 & Nova Lima/MG & Dias (2014) \\
\hline & & 10,86 & São Paulo/SP & Dias (2014) \\
\hline & & $1.222,58$ & Caratinga/MG & Sanson (2012) \\
\hline & & $13.440,00$ & Campinas/SP & Montagner \& Jardim (2011) \\
\hline \multirow{7}{*}{ Naproxeno } & & $1.090,00$ & Curitiba/PR & Goulart (2017) \\
\hline & & 145,00 & São Paulo/SP & Sousa et al. (2018) \\
\hline & & $2.455,00$ & São Carlos/SP & Campanha et al. (2014) \\
\hline & & 61,81 & Nova Lima/MG & \multirow{3}{*}{ Dias (2014) } \\
\hline & & 30,86 & Nova Iguaçu/RJ & \\
\hline & & 13,06 & São Paulo/SP & \\
\hline & & 404,71 & Goiânia/GO & Fonseca (2013) \\
\hline \multirow{7}{*}{ Diclofenaco } & \multirow{7}{*}{ Fármaco } & 470,00 & Curitiba/PR & Goulart (2017) \\
\hline & & 364,00 & São Paulo/SP & Sousa et al. (2018) \\
\hline & & 475,00 & São Carlos/SP & Campanha et al. (2014) \\
\hline & & 10,15 & Nova Lima/MG & \multirow{3}{*}{ Dias (2014) } \\
\hline & & 241,80 & Nova Iguaçu/RJ & \\
\hline & & 12,05 & São Paulo/SP & \\
\hline & & 165,15 & Goiânia/GO & Fonseca (2013) \\
\hline \multirow{8}{*}{ Quadro 2. Conti } & \multirow{8}{*}{ Estimulante } & $1.040,00$ & -MS & Sposito et al. (2018) \\
\hline & & $24.961,00$ & São Paulo/SP & Sousa et al. (2018) \\
\hline & & $3.167,00$ & Rio Doce/MG & Rodrigues et al. (2014) \\
\hline & & $474.790,00$ & São Carlos/SP & Campanha et al. (2014) \\
\hline & & $8.892,12$ & Goiânia/GO & Fonseca (2013) \\
\hline & & $3.010,32$ & Caratinga/MG & Sanson (2012) \\
\hline & & $127.092,00$ & Campinas/SP & Montagner \& Jardim (2011) \\
\hline & & $753.500,00$ & Curitiba/PR & Froehner et al. (2011) \\
\hline \multirow{3}{*}{ Atrazina } & \multirow{3}{*}{ Agrotóxico } & 171,30 & $-\mathrm{MS}$ & Sposito et al. (2018) \\
\hline & & $10.400,00$ & $-\mathrm{SP}$ & Santos (2013) \\
\hline & & 302,00 & $-\mathrm{PR}$ & Moura (2013) \\
\hline
\end{tabular}




\begin{tabular}{|c|c|c|c|c|}
\hline Composto & Classe & $\begin{array}{l}\text { Concentração } \\
\text { máxima }\left(n g L^{-1}\right)\end{array}$ & Localidade/Estado & Referência \\
\hline & & $4.920,00$ & -MT & Moreira et al. (2012) \\
\hline & & 611,00 & Campinas/SP & Raimundo (2011) \\
\hline \multirow{4}{*}{$\begin{array}{c}\text { Ácido } \\
\text { Acetilsalicílic } \\
\text { o (AAS) }\end{array}$} & \multirow{4}{*}{ Fármaco } & $1.650,00$ & Curitiba/PR & Goulart (2017) \\
\hline & & $4.761,27$ & Nova Lima/MG & \multirow{3}{*}{ Dias (2014) } \\
\hline & & $15.687,13$ & Nova Iguaçu/RJ & \\
\hline & & 299,50 & São Paulo/SP & \\
\hline \multirow{6}{*}{ Bisfenol A } & \multirow{6}{*}{ Plastificante } & 48,70 & -MS & Sposito et al. (2018) \\
\hline & & 75,34 & Rio Doce/MG & Rodrigues et al. (2014) \\
\hline & & 308,56 & Nova Lima/MG & \multirow{3}{*}{ Dias (2014) } \\
\hline & & 652,01 & Nova Iguaçu/RJ & \\
\hline & & 103,52 & São Paulo/SP & \\
\hline & & $13.016,00$ & Campinas/SP & Montagner \& Jardim (2011) \\
\hline \multirow{6}{*}{$\begin{array}{c}17-\alpha- \\
\text { etinilestradio } \\
1\end{array}$} & \multirow{6}{*}{ Hormônio } & 38,50 & -MS & Sposito et al. (2018) \\
\hline & & 0,53 & Rio Doce/MG & Rodrigues et al. (2014) \\
\hline & & 236,63 & Nova Iguaçu/RJ & \multirow{3}{*}{ Dias (2014) } \\
\hline & & 138,16 & São Paulo/SP & \\
\hline & & 45,33 & Nova Lima/MG & \\
\hline & & $9.520,00$ & Curitiba/PR & Padilha \& Leitzke (2013) \\
\hline \multirow{4}{*}{ Atenolol } & \multirow{30}{*}{ Fármaco } & 665,00 & São Paulo/SP & Sousa et al. (2018) \\
\hline & & $66.136,00$ & São Carlos/SP & Campanha et al. (2014) \\
\hline & & $1.627,62$ & Catas Altas/MG & Quaresma (2014) \\
\hline & & 961,08 & Goiânia/GO & Fonseca (2013) \\
\hline \multirow{6}{*}{ Triclosan } & & 8,60 & -MS & Sposito et al. (2018) \\
\hline & & 61,30 & São Paulo/SP & Sousa et al. (2018) \\
\hline & & 66,00 & São Paulo/SP & Montagner et al. (2014) \\
\hline & & 281,10 & São Carlos/SP & Campanha et al. (2014) \\
\hline & & 82,43 & Goiânia/GO & Fonseca (2013) \\
\hline & & 66,00 & Campinas/SP & Montagner \& Jardim (2011) \\
\hline \multirow{3}{*}{ Genfibrozila } & & 210,06 & Nova Lima/MG & \multirow{12}{*}{ Dias (2014) } \\
\hline & & 200,32 & Nova Iguaçu/RJ & \\
\hline & & 10,37 & São Paulo/SP & \\
\hline \multirow{3}{*}{$\begin{array}{c}\text { Trimetoprim } \\
\text { a }\end{array}$} & & $1.573,86$ & São Paulo/SP & \\
\hline & & $1.409,06$ & Nova Iguaçu/RJ & \\
\hline & & 624,60 & Nova Lima/MG & \\
\hline \multirow{3}{*}{ Octilfenol } & & 50,27 & Nova Lima/MG & \\
\hline & & 37,56 & Nova Iguaçu/RJ & \\
\hline & & 16,04 & São Paulo/SP & \\
\hline \multirow{3}{*}{ Quakpfol fercodnt } & & $1.914,30$ & Nova Lima/MG & \\
\hline & & 98,04 & São Paulo/SP & \\
\hline & & 38,23 & Nova Iguaçu/RJ & \\
\hline \multirow{3}{*}{$\begin{array}{c}\text { Carbamazepi } \\
\text { na }\end{array}$} & & 659,00 & São Paulo/SP & Sousa et al. (2018) \\
\hline & & 452,50 & São Carlos/SP & Campanha et al. (2014) \\
\hline & & 84,10 & Goiânia/GO & Fonseca (2013) \\
\hline Benzafibrato & & 744,41 & Nova Iguaçu/RJ & \multirow{2}{*}{ Dias (2014) } \\
\hline & & 554,30 & Nova Lima/MG & \\
\hline \multirow{3}{*}{ Propanolol } & & 48,10 & São Paulo/SP & Sousa et al. (2018) \\
\hline & & $1.528,00$ & São Carlos/SP & Campanha et al. (2014) \\
\hline & & 21,84 & Goiânia/GO & Fonseca (2013) \\
\hline
\end{tabular}




\begin{tabular}{|c|c|c|c|c|}
\hline Composto & Classe & $\begin{array}{c}\text { Concentração } \\
\text { máxima (ng L-1) }\end{array}$ & Localidade/Estado & Referência \\
\hline \multirow{3}{*}{ Estriol } & \multirow{3}{*}{ Hormônio } & 11,90 & $-\mathrm{MS}$ & Sposito et al. (2018) \\
\hline & & 67,39 & Nova Lima/MG & \multirow{2}{*}{ Dias (2014) } \\
\hline & & 15,81 & São Paulo/SP & \\
\hline \multirow{2}{*}{ Progesterona } & \multirow{2}{*}{ Hormônio } & 530,00 & Curitiba/PR & Goulart (2017) \\
\hline & & 195,00 & Campinas/SP & Montagner \& Jardim (2011) \\
\hline Carbendazim & \multirow{6}{*}{ Agrotóxico } & 96,00 & \multirow{6}{*}{ MS } & \multirow{6}{*}{ Sposito et al. (2018) } \\
\hline Hexazinona & & 16,00 & & \\
\hline Diuron & & 11,70 & & \\
\hline $\begin{array}{c}\text { Imidacloprid } \\
\mathrm{a}\end{array}$ & & 53,50 & & \\
\hline Tebuthiuron & & 44,10 & & \\
\hline Fipronil & & 29,20 & & \\
\hline $\begin{array}{c}\text { Ácido } \\
\text { salicílico }\end{array}$ & \multirow{4}{*}{ Fármaco } & $31.850,00$ & \multirow[t]{2}{*}{ Curitiba/PR } & \multirow[t]{2}{*}{ Goulart (2017) } \\
\hline Cetoprofeno & & 260,00 & & \\
\hline Azitromicina & & $8.172,61$ & Frei Inocêncio/MG & Quaresma (2014) \\
\hline $\begin{array}{l}\text { Sulfametoxaz } \\
\text { ol }\end{array}$ & & $1.826,30$ & Nova Lima/MG & Dias (2014) \\
\hline Cocaína & \multirow[b]{2}{*}{ Droga ilícita } & $5.896,00$ & \multirow[b]{2}{*}{ Manaus/AM } & \multirow[b]{2}{*}{ Thomas et al. (2014) } \\
\hline $\begin{array}{l}\text { Benzoilecgon } \\
\text { ina }\end{array}$ & & $3.582,00$ & & \\
\hline
\end{tabular}

As concentrações de estrona, $17 \beta$-estradiol, estriol e 17- $\alpha$-etinilestradiol foram superiores no Brasil, em comparação com os países como Canadá, China, Reino Unido, França, Alemanha e Grécia (Kleywegt et al., 2011; Peng et al., 2008; Kasprzyk-Hordern et al., 2009; Vulliet \& Cren-Olivé, 2011; Regnery \& Puttmann 2010; Stasinakis et al., 2013). Enquanto as concentrações destes estrogênios no cenário internacional não ultrapassaram o valor de 72,60 $\mathrm{ng} \mathrm{L}^{-1}$, no Brasil chegaram a 9.520,00 ng L-1 em águas superficiais.

Em relação a cafeína, AAS e atrazina, que foram alguns dos CPEs encontrados em maiores concentrações, atingindo concentrações máximas médias de $174.557,00 \mathrm{ng} \mathrm{L}^{-1}, 5.600,00 \mathrm{ng} \mathrm{L}^{-1}$ e 3.281,00 $\mathrm{ng} \mathrm{L}^{-1}$, respectivamente, estudos internacionais demonstraram que as concentrações desses três compostos foram inferiores aos do Brasil. As concentrações de cafeína variaram de 0 a 1.894,10 ng L-1 na França, Alemanha e Grécia (Vulliet \& Cren-Olivé, 2011; Regnery \& Puttmann, 2010; Stasinakis et al., 2013). Já para o AAS e atrazina, Collado et al. (2014), Vulliet \& Cren-Olivé (2011), Regnery \& Puttmann (2010) e Stasinakis et al. (2013) não detectaram a presença desses dois compostos nas águas superficiais da Espanha, França, Alemanha e Grécia, respectivamente.

Provavelmente esses resultados se devem às melhores condições de saneamento encontradas e aos sistemas utilizados no tratamento de efluentes mais eficientes. Contudo, deve-se levar em conta as diferenças de datas desses trabalhos, além de diferenças populacionais e demográficas, acarretando diferenças das concentrações dos CPEs encontrados.

\section{ÁGUA POTÁVEL}

Percebe-se que há um maior número de dados de monitoramento de CPEs em água superficial (potenciais mananciais de abastecimento) em comparação à água distribuída para a população. Sobre a ocorrência dos CPEs em águas potáveis no Brasil, foram encontrados 8 trabalhos que avaliaram a presença de 31 compostos em treze estados (Rio de Janeiro, São Paulo, Minas Gerais, Paraná, Rio Grande do Sul, Piauí, Pará, Mato Grosso, Espírito Santo, Mato Grosso do Sul, Paraíba, Ceará e Santa Catarina).

O Quadro 3 mostra que a presença de CPEs em água para abastecimento humano no Brasil, é uma realidade, uma vez que foram encontrados 31 compostos nesta matriz, sendo 15 compostos 
pertencente a classe dos fármacos, 7 agrotóxicos, 4 compostos na classe dos hormônios, 2 plastificantes, 2 compostos industriais, e 1 estimulante.

No estudo de Dias (2014), 16 microcontaminantes foram detectados nos sistemas de abastecimento de água das regiões metropolitanas de Belo Horizonte, Rio de Janeiro e São Paulo, comprovando a presença desses poluentes em mananciais que abastecem cerca de 15 milhões de pessoas. Dias (2014) ainda recomenda um maior monitoramento de mananciais brasileiros a fim de aumentar o número de dados sobre a ocorrência de interferentes endócrinos e fármacos em sistemas de abastecimento de água, bem como o desenvolvimento de novas técnicas de remoção de microcontaminantes de preocupação emergente nos tratamentos de água e esgoto.

Quadro 3. Concentrações de contaminantes de preocupação emergente determinados em água potável no Brasil (VMP)

\begin{tabular}{|c|c|c|c|c|}
\hline Composto & Classe & $\begin{array}{c}\text { Concentração } \\
\text { máxima } \\
\left(n g L^{-1}\right)\end{array}$ & Localidade/Estado & Referência \\
\hline \multirow{12}{*}{ Atrazina } & \multirow{12}{*}{ Agrotóxico } & 24,00 & Belo Horizonte/MG & \multirow{9}{*}{$\begin{array}{l}\text { Machado et al. } \\
\quad(2016)\end{array}$} \\
\hline & & 15,00 & Florianópolis/SC & \\
\hline & & 15,00 & Fortaleza/CE & \\
\hline & & 15,00 & João Pessoa/PB & \\
\hline & & 15,00 & Teresina/PI & \\
\hline & & 15,00 & São Paulo/SP & \\
\hline & & 15,00 & Vitória/ES & \\
\hline & & 15,00 & Cuiabá/MT & \\
\hline & & 15,00 & Campo Grande/MS & \\
\hline & & 10,00 & Curitiba/PR & Machado (2015) \\
\hline & & 92,30 & Morro Redondo/RS & Caldas et al. (2013) \\
\hline & & 687,00 & Campinas/SP & Raimundo (2011) \\
\hline \multirow{11}{*}{ Cafeína } & \multirow{11}{*}{ Estimulante } & $2.769,00$ & Porto Alegre/RS & \multirow{9}{*}{$\begin{array}{l}\text { Machado et al. } \\
\text { (2016) }\end{array}$} \\
\hline & & $1.793,00$ & Campo Grande/MS & \\
\hline & & 167,00 & Curitiba/PR & \\
\hline & & 348,00 & São Paulo/SP & \\
\hline & & 267,00 & Vitória/ES & \\
\hline & & 599,00 & Belo Horizonte/MG & \\
\hline & & 629,00 & Cuiabá/MT & \\
\hline & & 133,00 & Belém/PA & \\
\hline & & 196,00 & Teresina/PI & \\
\hline & & 167,00 & Curitiba/PR & Machado (2015) \\
\hline & & $4.324,00$ & Campinas/SP & Raimundo (2011) \\
\hline \multicolumn{2}{|c|}{ Quadro 3. Continuação... } & 43,45 & Nova Lima/MG & \multirow{3}{*}{ Dias (2014) } \\
\hline \multirow{3}{*}{$17 \beta$-estradiol } & \multirow{7}{*}{ Hormônio } & 17,28 & Nova Iguaçu/RJ & \\
\hline & & 40,52 & São Paulo/SP & \\
\hline & & 6,80 & Jaboticabal/SP & Lopes et al. (2010) \\
\hline \multirow{4}{*}{ Estrona } & & 70,07 & Nova Lima/MG & \multirow{3}{*}{ Dias (2014) } \\
\hline & & 58,35 & Nova Iguaçu/RJ & \\
\hline & & 17,31 & São Paulo/SP & \\
\hline & & 5,00 & Campinas/SP & Raimundo (2011) \\
\hline \multirow{4}{*}{ Bisfenol A } & \multirow{5}{*}{ Plastificante } & 2549,14 & Nova Lima/MG & \multirow{3}{*}{ Dias (2014) } \\
\hline & & 224,93 & Nova Iguaçu/RJ & \\
\hline & & 127,44 & São Paulo/SP & \\
\hline & & 26,00 & Campinas/SP & Raimundo (2011) \\
\hline Dibutilftalato & & 175,00 & Guararema/SP & Souza et al. (2012) \\
\hline
\end{tabular}




\begin{tabular}{|c|c|c|c|c|}
\hline Composto & Classe & $\begin{array}{l}\text { Concentração } \\
\text { máxima } \\
\left(n g L^{-1}\right)\end{array}$ & Localidade/Estado & Referência \\
\hline & & 188,00 & São José dos Campos/SP & \\
\hline & & 247,00 & Taubaté/SP & \\
\hline & & 454,00 & Pindamonhangaba/SP & \\
\hline \multirow{3}{*}{ Dietilftalato } & \multirow{3}{*}{ Composto industrial } & 48,00 & São José dos Campos/SP & \multirow{3}{*}{ Souza et al. (2012) } \\
\hline & & 129,00 & Taubaté/SP & \\
\hline & & 143,00 & Pindamonhangaba/SP & \\
\hline \multirow{3}{*}{ AAS } & \multirow{3}{*}{ Fármaco } & $4.155,53$ & Nova Lima/MG & \multirow{3}{*}{ Dias (2014) } \\
\hline & & $5.286,94$ & Nova Iguaçu/RJ & \\
\hline & & 343,02 & São Paulo/SP & \\
\hline \multirow{3}{*}{$\begin{array}{c}17-\alpha- \\
\text { etinilestradiol }\end{array}$} & \multirow{3}{*}{ Hormônio } & 22,52 & Nova Lima/MG & \multirow{30}{*}{ Dias (2014) } \\
\hline & & 622,99 & Nova Iguaçu/RJ & \\
\hline & & 29,50 & São Paulo/SP & \\
\hline \multirow{3}{*}{ Ibuprofeno } & \multirow{25}{*}{ Fármaco } & 16,33 & Nova Lima/MG & \\
\hline & & 16,36 & Nova Iguaçu/RJ & \\
\hline & & 16,86 & São Paulo/SP & \\
\hline \multirow{3}{*}{ Diclofenaco } & & 330,58 & Nova Lima/MG & \\
\hline & & 101,01 & Nova Iguaçu/RJ & \\
\hline & & 38,09 & São Paulo/SP & \\
\hline \multirow{3}{*}{ Genfibrozila } & & 81,82 & Nova Lima/MG & \\
\hline & & 98,39 & Nova Iguaçu/RJ & \\
\hline & & 29,82 & São Paulo/SP & \\
\hline \multirow{3}{*}{ Naproxeno } & & 216,78 & Nova Lima/MG & \\
\hline & & 252,64 & Nova Iguaçu/RJ & \\
\hline & & 62,47 & São Paulo/SP & \\
\hline \multirow{3}{*}{ Trimetoprima } & & $1.781,00$ & Nova Iguaçu/RJ & \\
\hline & & $4.381,21$ & São Paulo/SP & \\
\hline & & 876,39 & Nova Lima/MG & \\
\hline \multirow{3}{*}{ Octilfenol } & & 276,58 & Nova Lima/MG & \\
\hline & & 45,66 & Nova Iguaçu/RJ & \\
\hline & & 8,17 & São Paulo/SP & \\
\hline \multirow{3}{*}{ Nonilfenol } & & 395,98 & Nova Lima/MG & \\
\hline & & 54,35 & Nova Iguaçu/RJ & \\
\hline & & $2.820,04$ & São Paulo/SP & \\
\hline \multirow{2}{*}{ Paracetamol } & & 453,60 & Nova Lima/MG & \\
\hline & & 421,64 & São Paulo/SP & \\
\hline \multirow{2}{*}{ Bezafibrato } & & $1.659,10$ & Nova Iguaçu/RJ & \\
\hline & & 64,62 & São Paulo/SP & \\
\hline \multirow{2}{*}{ Estriol } & \multirow{2}{*}{ Hormônio } & 59,29 & Nova Lima/MG & \\
\hline & & 97,39 & São Paulo/SP & \\
\hline Fenolftaleína & Composto industrial & 14,80 & \multirow{3}{*}{ Campinas/SP } & Raimundo(2011) \\
\hline Triclosan & \multirow{3}{*}{ Fármaco } & 37,00 & & Raimundo (2011) \\
\hline Amoxilina & & 8,90 & & Locatelli et al. (2011) \\
\hline Sulfametoxazol & & $2.592,60$ & Nova Lima/MG & Dias (2014) \\
\hline Carbofurano & & 23,80 & & \\
\hline Clomazona & & 123,90 & & \\
\hline Diuron & Agrotóxico & 95,80 & Morro Redondo/RS & Caldas et al. (2013) \\
\hline Epoxiconazol & & 83,00 & & \\
\hline
\end{tabular}




\begin{tabular}{|c|c|c|c|c|}
\hline Composto & Classe & $\begin{array}{c}\text { Concentração } \\
\text { máxima } \\
\left(n g L^{-1}\right)\end{array}$ & Localidade/Estado & Referência \\
\hline Irgarol & & 7,20 & & \\
\hline Tebuconazol & & 76,70 & & \\
\hline Mebendazol & \multirow{2}{*}{ Fármaco } & 18,50 & & \\
\hline Propilparabeno & & 135,50 & & \\
\hline
\end{tabular}

Machado et al. (2016) avaliaram a presença de CPEs em cem amostras coletadas em 22 capitais brasileiras. Cafeína e atrazina foram as substâncias mais frequentemente detectadas na água potável. Foi detectada atrazina, a uma concentração de pelo menos 15,00 ng $\mathrm{L}^{-1}$, em amostras coletadas em Florianópolis, Fortaleza, João Pessoa, Teresina, São Paulo, Vitória, Cuiabá e Campo Grande.

Machado (2015) menciona que a ampla presença de cafeína nas amostras de água tratada, por se tratar de um composto de origem antrópica, é um indício da contaminação do manancial principalmente por esgoto doméstico. Ainda que a maioria dos compostos não sejam legislados, o conhecimento acerca do comportamento e concentrações dessas substâncias em água é de suma importância, uma vez que ainda não se conhece as doses seguras de ingestão para inúmeros destes compostos.

As concentrações de ibuprofeno, sulfametazol e bisfenol A observados por Simazaki et al. (2015) em água tratada para o consumo humano no Japão, apresentaram concentrações máximas inferiores a 100,00 ng L-1, em média. Com exceção do ibuprofeno, que também se manteve inferior a 100,00 ng L $\mathrm{L}^{-1}$, as concentrações encontradas no Brasil para os outros dois CPEs foram superiores aos encontrados no Japão.

Um ponto que merece atenção dentre os estudos de âmbito nacional, é ampla dispersão das concentrações, nas três matrizes estudadas. Os diferentes autores detectaram concentrações em ordens de grandeza bem distintas entre si, o que dificulta o estabelecimento de concentrações médias desses compostos para cada uma das matrizes. Tais variações podem ser explicadas por diversos fatores, como: condições locais, diferenças sociais e de saúde pública, e, ainda, diferenças entre as metodologias aplicadas para a condução de cada um dos estudos (Xu et al., 2012).

Raimundo (2011) destaca que, como não há uma legislação ambiental, até o momento, que inclua os CPEs, a apresentação do problema fornece subsídios reais para que as agências regulatórias e órgãos do governo possam direcionar pesquisas e discussões no sentido de estabelecer os melhores padrões para a preservação da vida aquática, conservação dos mananciais e a potabilidade da água distribuída para a população.

\section{DINÂMICA DOS CPES NOS AMBIENTES AQUÁTICOS}

Alguns dos CPEs mais relatados na literatura brasileira foram selecionados para uma análise de suas dinâmicas nos ambientes aquáticos abordados neste artigo. As Figuras 3, 4 e 5 apresentam as concentrações máximas de $17 \beta$-estradiol, diclofenaco, bisfenol A, estrona e atrazina respectivamente, quando determinadas nas amostras de esgoto doméstico bruto e tratado, bem como nos corpos hídricos superficiais e água tratada, de acordo com os dados descritos nos Quadros 1, 2 e 3. Dentre os compostos estudados nestas matrizes, o hormônio $17 \beta$-estradiol foi o mais frequentemente investigado nas matrizes de esgoto bruto e tratado e na matriz de águas superficiais. Já para a matriz de água potável, os CPEs atrazina e cafeína foram os mais frequentemente estudados.

Observa-se na Figura 3a que os valores encontrados no esgoto tratado foram pouco inferiores aos encontrados no esgoto bruto, o que sugere a remoção de parte desses compostos nas ETEs. 0 fato de terem sido relatadas concentrações nos rios mais altas que no esgoto bruto, pode sugerir que os esgotos não são a principal fonte poluidora dos rios para este composto. É válido ressaltar que o número de estudos considerados nas duas matrizes pode influenciar nos resultados obtidos.

No caso do diclofenaco (Figura 3b), as concentrações relatadas para as águas superficiais e tratadas foram da mesma ordem de grandeza, sugerindo a baixa eficiência das ETAs na remoção desse composto. Ressalta-se que para o esgoto tratado, não foram encontrados trabalhos que avaliassem a ocorrência do diclofenaco.

No caso do bisfenol A (Figura 4a), percebe-se menores concentrações nos efluentes das ETEs em relação às dos esgotos brutos, sugerindo a eficiência de remoção deste composto nas ETEs. Já em relação as águas superficiais e tratadas, a concentração detectada na água potável foi superior que 
aquela observada nos corpos hídricos superficiais. Ressalta-se que houve um valor máximo para esse CPE de 13.016,00 ng L-1 (Montagner \& Jardim, 2011) para a matriz de água superficial, que foi considerado como outlier e não foi plotado na Figura 4, para melhor visualização dos demais valores.

Para o hormônio estrona (Figura 4b), percebe-se que as concentrações encontradas em esgotos tratados foram inferiores quando comparados ao esgoto bruto, sugerindo a remoção de parte desse composto pelas tecnologias adotadas nas ETEs. Já em relação as águas superficiais e tratadas, assim como o bisfenol A, a concentração de estrona detectada na água potável foi da mesma ordem de grandeza que aquela observada nos corpos hídricos superficiais, e a compilação feita neste estudo sugere que tais compostos não são eficientemente removidos pelos processos clássicos de clarificação e desinfecção utilizados nas ETAs.

Em relação a atrazina (Figura 5), observa-se que as concentrações encontradas em águas superficiais foram superiores às encontradas em água potável, mostrando possível degradação desse composto pelas ETAs. Ressalta-se que não foram encontrados trabalhos que avaliassem a ocorrência de atrazina em esgotos brutos e tratados.
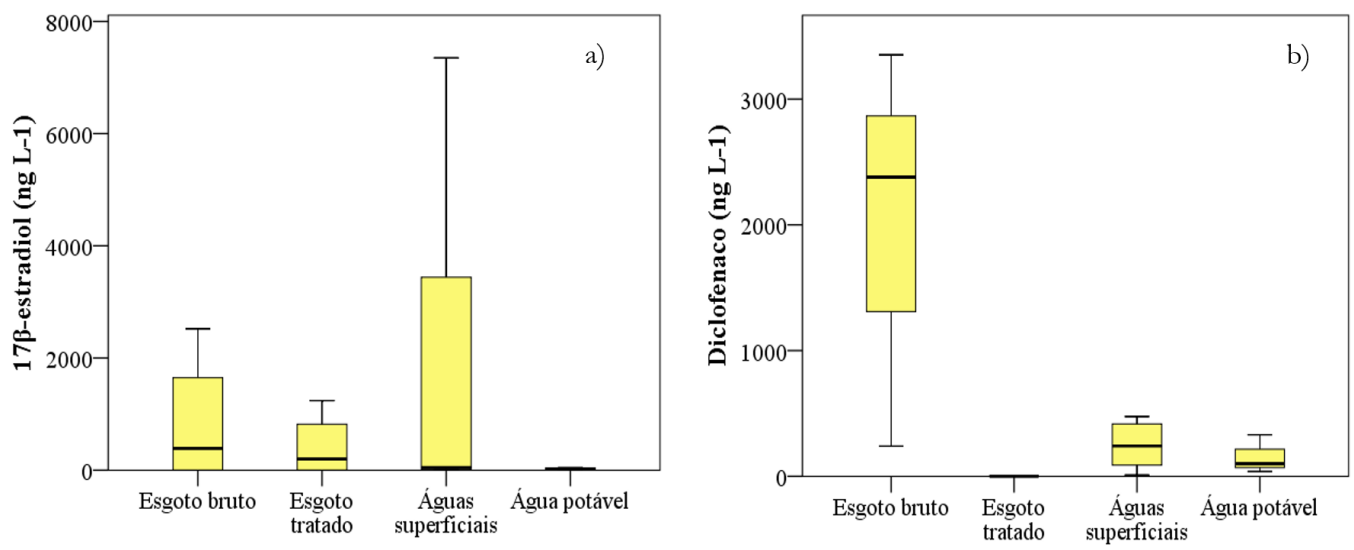

Figura 3. Boxplot das concentrações máximas de (a) 17 $\beta$-estradiol e (b) diclofenaco determinados nas matrizes aquáticas no Brasil de acordo com os dados apresentados nos Quadros 1, 2 e 3
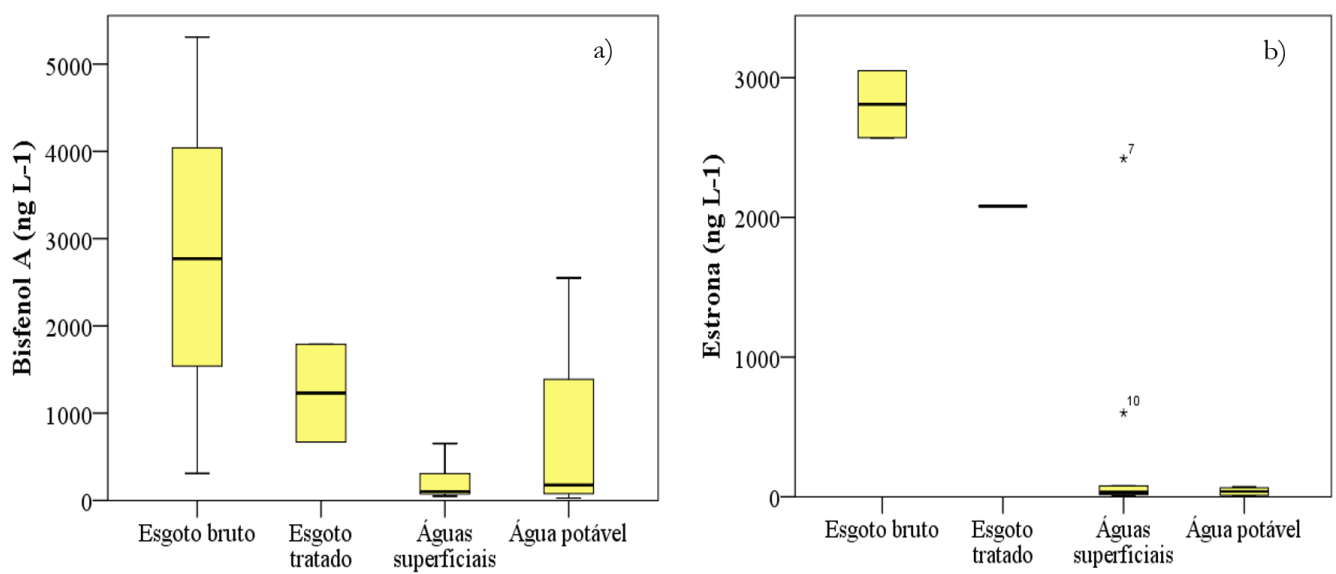

Figura 4. Boxplot das concentrações máximas de (a) bisfenol A e (b) estrona determinados nas matrizes aquáticas no Brasil de acordo com os dados apresentados nos Quadros 1, 2 e 3 


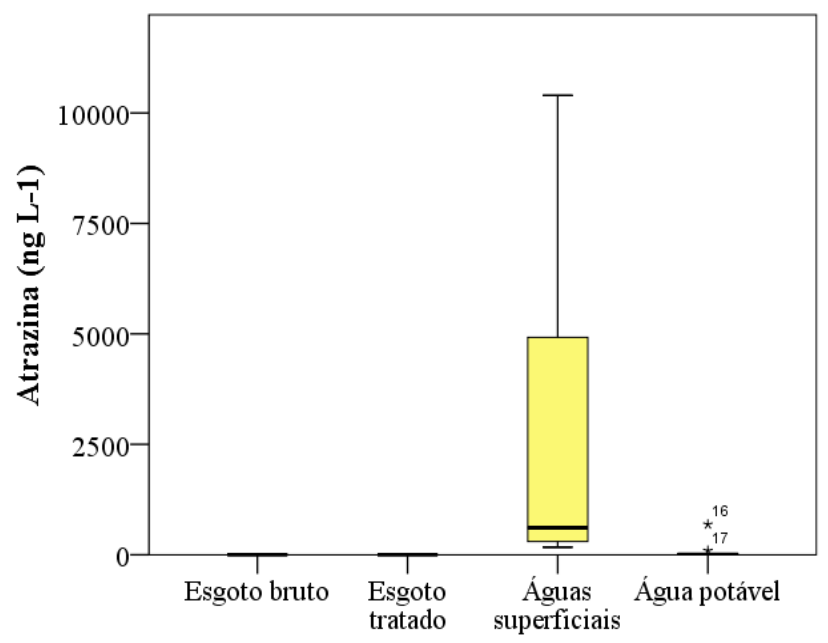

Figura 5. Boxplot das concentrações máximas de Atrazina determinados nas matrizes aquáticas no Brasil de acordo com os dados apresentados nos Quadros 1, 2 e 3

De acordo com Lima et al. (2017), uma vez lançado nos recursos hídricos, determinados CPEs podem ter sua concentração reduzida por mecanismos naturais de remoção, tais como hidrólise (reação de natureza não redox com a água), volatilização (passagem para a fase gasosa), adsorção (retenção na superfície de sólidos), absorção (encapsulamento, por exemplo, por gotas de óleo), oxidação ou redução (reação com espécies de elevado potencial de redução ou oxidação, respectivamente) ou fotólise (degradação mediada pela radiação solar). Dessa forma, de acordo com os mesmos autores, a análise do destino de um CPE no meio hídrico passa pelo conhecimento das suas principais características e propriedades físico-químicas, o que contribui, ainda, para o melhor entendimento do comportamento desses em estações de tratamento de águas. Ademais, foi possível quantificar contaminantes de preocupação emergente em todas as matrizes aquáticas, inclusive na água tratada, o que remete a fragilidade do saneamento básico e aos elevados índices de consumo do país.

\section{CONSIDERAÇÕES FINAIS}

Os contaminantes de preocupação emergente são uma realidade presente em águas do território brasileiro, podendo ocorrer um decréscimo em suas concentrações ao longo da seguinte sequência de ambientes aquáticos: esgoto bruto, esgoto tratado, águas superficiais (rios) e águas de abastecimento público (ETA).

As concentrações de CPEs mais baixas encontradas nos efluentes das ETEs em relação aos esgotos brutos sugerem que as estações de tratamento de esgotos utilizadas no Brasil apresentam alguma eficiência de remoção para estes compostos, embora de magnitude reduzida. Para uma melhoria do cenário dos CPEs em águas superficiais e de ETAs, um aumento no percentual de esgoto tratado no Brasil é essencial.

Dos 5 CPEs objeto de comparação, o $17 \beta$-estradiol e a atrazina apresentaram concentrações maiores em águas superficiais em relação à tratada. Entretanto, a concentração de diclofenaco, estrona e bisfenol A detectados na água potável foi da mesma ordem de grandeza que aquela observada nos corpos hídricos superficiais, e a compilação feita neste estudo sugere que tais compostos não são eficientemente removidos pelos processos clássicos de clarificação e desinfecção utilizados nas ETAs. Desta forma, para uma melhoria do cenário dos CPEs em águas de abastecimento, para alguns dos compostos, técnicas especiais de tratamento de água devem ser consideradas.

Com relação a comparação dos cenários nacional e internacional, para a matriz de esgoto bruto e tratado, o cenário se mostrou semelhante. Contudo, para as águas superficiais e potável, a concentração dos CPEs no Brasil se apresentou superior ao cenário internacional.

\section{REFERÊNCIAS}

Agência Nacional de Vigilância Sanitária - ANVISA. (2018). Recuperado em 25 de setembro de 2019, de http://portal.anvisa.gov.br/documents/111215/117782/a14.pdf/76773817-be41-4334-9846$77 \mathrm{bd} 559 f 9 \mathrm{e} 80$ 
Alves, T. C., Girardi, R., \& Pinheiro, A. (2017). Micropoluentes orgânicos: ocorrência, remoção e regulamentação. REGA, 14, e1.

Aquino, S. F., Brandt, E. M. F., \& Chernicharo, C. A. L. (2013). Remoção de fármacos e desreguladores endócrinos em estações de tratamento de esgoto: revisão da literatura. Engenharia Sanitaria e Ambiental, 18(3), 187204.

Araújo, R. K., Wolff, D. B., \& Carissimi, E. (2019). Fármacos em águas residuárias: efeitos ambientais e remoção em wetlands construídos. Revista DAE, 67(218), 137-155.

Bahlmann, A., Brack, W., Schneider, R. J., \& Krauss, M. (2014). Carbamazepine and its metabolites in wastewater: analytical pitfalls and occurrence in Germany and Portugal. Water Research, 57, 104-114.

Bila, D. M., \& Dezzotti, M. (2007). Desreguladores endócrinos no meio ambiente: efeitos e consequências. Quimica Nova, 30, 651-666.

Brasil. Ministério do Meio Ambiente. (2005). Resolução CONAMA no 357, de 15 de junho de 2005. Dispõe sobre a classificação dos corpos de água e diretrizes ambientais para o seu enquadramento, bem como estabelece as condições e padrões de lançamento de efluentes, e dá outras providências. Diário Oficial [da] República Federativa do Brasil, Brasília.

Caldas, S. S., Bolzan, C. M., Guilherme, J. R., Silveira, M. A. K., Escarrone, A. L. V., \& Primel, E. G. (2013). Determination of pharmaceuticals, personal care products, and pesticides in surface and treated waters: method development and survey. Environmental Science and Pollution Research International, 20, 58555863.

Campanha, M. B., Awan, A. T., De Sousa, D. N. R., Grosseli, G. M., Mozeto, A. A., \& Fadini, P. S. (2014). A 3-year study on occurrence of emerging contaminants in an urban stream of São Paulo State of Southeast Brazil. Environmental Science and Pollution Research International, 22(10), 7936-7947.

Collado, N., Rodriguez-Mozaz, S., Gros, M., Rubirola, A., Barceló, D., Comas, J., Rodriguez-Roda, I., \& Buttiglieri, G. (2014). Pharmaceuticals occurrence in a WWTP with significant industrial contribution and its input into the river system. Environmental Pollution, 185, 202-212.

Crain, D. A., Janssen, S. J., Edwards, T. M., Heindel, J., Ho, S., Hunt, P., Iguchi, T., Juul, A., Mclachalan, J. A., Schwartz, J., Skakkebaek, N., Soto, A. M., Swan, S., Walker, C., Woodruff, T. K., Woodruff, T. J., Giudice, L. C., \& Guillette Junior, L. J. (2008). Female reproductive disorders: the roles of endocrine-disrupting compounds and developmental timing. Fertility and Sterility, 90(4)

De Paiva Pessoa, G., Dos Santos, A. B., De Souza, N. C., Alves, J. A. C., \& Do Nascimento, R. F. (2012). Desenvolvimento de metodologia para avaliar remoção de estrogênios em estações de tratamento de esgotos. Química Nova, 35(5), 968-973.

Dias, R. V. A. (2014). Avaliação da ocorrência de microcontaminantes de preocupação emergentes em sistemas de abastecimento de água e da atividade estrogênica do estinilestradiol (Dissertação de mestrado). Universidade Federal de Minas Gerais, Belo Horizonte.

Du, B., Price, A. E., Scott, W. C., Kristofco, L. A., Ramirez, A. J., Chambliss, C. K., Yelderman, J. C., \& Brooks, B. W. (2014). Comparison of contaminants of emerging concern removal, discharge, and water quality hazards among centralized and on-site wastewater treatment system effluents receiving common wastewater influent. The Science of the Total Environment, 466-467, 976-984.

Fairbairn, D. J., Karpuzcu, M. E., Arnold, W. A., Barber, B. L., Kaufenberg, E. F., Koskinen, W. C., Novak, P. J., Rice, P. J., \& Swackhamer, D. L. (2016). Sources and transport of contaminants of emerging concern: A two-year study of occurrence and spatiotemporal variation in a mixed land use watershed. The Science of the Total

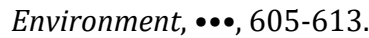

Ferreira, A. P. (2013). Desreguladores endócrinos em estações de tratamento de esgotos: complicações ao meio ambiente. Acta Scientiarum. Technology, 35(2), 307-316.

Fonseca, Y. V. P. (2013). Estudo sobre a ocorrência de poluentes emergentes na água do Rio Meia Ponte na cidade de Goiânia-GO (Dissertação de mestrado). Universidade Federal de Goiás, Goiânia.

Froehner, S., Machado, K. S., Stefen, E., \& Nolasco, M. (2011). Occurrence of sexual hormones in sediments of mangrove in Brazil. Water, Air, and Soil Pollution, 219(1), 591-599.

Gavrilescu, M., Demnerová, K., Aamand, J., Agathos, S., \& Fava, F. (2015). Emerging pollutants in the environment: present and future challenges in biomonitoring, ecological risks and bioremediation. New Biotechnology, 32(1), 147-156. 
Goulart, F. A. B. (2017). Contaminantes emergentes em um país emergente: estudo de caso no Rio Barigui (Dissertação de mestrado). Programa de Pós-graduação em Ciência e Tecnologia Ambiental, Universidade Tecnológica Federal do Paraná, Curitiba.

Grosseli, M. G. (2016). Contaminantes emergentes em estações de tratamento de esgoto aeróbia e anaeróbia (Tese de doutorado). Centro de Ciências e de Tecnologia, Universidade Federal de São Carlos, São Carlos.

Instituto Brasileiro do Meio Ambiente - IBAMA. (2018). Recuperado em 25 de setembro de 2019, de http://www.ibama.gov.br/agrotoxicos/relatorios-de-comercializacao-de-agrotoxicos

Jelic, A., Gros, M., Ginebreda, A., Cespedes-Sánchez, R., Ventura, F., Petrovic, M., \& Barcelo, D. (2011). Occurrence, partition and removal of pharmaceuticals in sewage water and sludge during wastewater treatment. Water Research, 45, 1165-1176.

Kasprzyk-Hordern, B., Dinsdale, R. M., \& Guwy, A. J. (2009). The removal of pharmaceuticals, personal care products, endocrine disruptors and illicit drugs during wastewater treatment and its impact on the quality of receiving waters. Water Research, 43(2), 363-380.

Kleywegt, S., Pileggi, V., Yang, P., Hao, C., Zhao, X., Rocks, C., Thach, S., Cheung, P., \& Whitehead, B. (2011). Pharmaceuticals, hormones and bisphenol A in untreated source and finished drinking water in Ontario, Canada - Occurrence and treatment efficiency. The Science of the Total Environment, 409(8), 1481-1488.

Lee, H. B., Peart, T. E., \& Svoboda, M. L. (2005). Determination of endocrine-disrupting phenols, acidic pharmaceuticals, and personal-care products in sewage by solid-phase extraction and gas chromatography - mass spectrometry. Journal of Chromatography. A, 1094(1-2), 122-129.

Lima, D. R. S., Tonucci, M. C., Libânio, M., \& Aquino, S. F. (2017). Fármacos e desreguladores endócrinos em águas brasileiras: ocorrência e técnicas de remoção. Engenharia Sanitaria e Ambiental, 22(6), 1043-1054.

Locatelli, M. A. F., Sodre, F. F., \& Jardim, W. F. (2011). Determination of antibiotics in Brazilian surface waters using liquid chromatography-electrospray tandem mass spectrometry. Archives of Environmental Contamination and Toxicology, 60(3), 385-393.

Lopes, L. G., Marchi, M. R. R., Souza, J. B. G., Moura, J. A., Lorenzon, C. S., Cruz, C., \& Amaral, L. A. (2010). Estrogênios em águas naturais e tratadas da região de Jaboticabal - São Paulo. Quimica Nova, 33, 639.

Luo, Y., Guo, W., Ngo, H. H., Nghiem, L. D., \& Hai, F. I., Zhang, J., Liang, S., \& Wang, X. C. (2014). A review on the occurrence of micropollutants in the aquatic environment and their fate and removal during wastewater treatment. The Science of the Total Environment, 473-474, 619-641.

Machado, G. C. (2015). Contaminantes químicos emergentes em águas naturais e de abastecimento público: desenvolvimento analítico e estudo de caso envolvendo capitais estaduais do Brasil (Tese de doutorado). Universidade Federal do Paraná, Curitiba.

Machado, K. C., Grassi, M. T., Vidal, C., Pescara, I. C., Jardim, W. F., Fernandes, A. N., Sodré, F. F., Almeida, F. V., Santana, J. S., Canela, M. C., Nunes, C. R. O., Bichinho, K. M., \& Severo, F. J. R. (2016). A preliminary nationwide survey of the presence of emerging contaminants in drinking and source waters in Brazil. The Science of the Total Environment, 572, 138-146.

Maldaner, A. O., Schmidt, L. L., Locatelli, M. A. F., Jardim, W. F., Sodré, F. F., Almeida, F. V., Pereira, C. E. B., \& Silva, C. M. S. (2012). Estimating Cocaine Consumption in the Brazilian Federal District (FD) by Sewage Analysis. Journal of the Brazilian Chemical Society, 23(5), 861-867.

Montagner, C. C., \& Jardim, W. F. (2011). Spatial and seasonal variations of pharmaceuticals and endocrine disruptors in the Atibaia River, São Paulo State (Brazil). Journal of the Brazilian Chemical Society, 22(8), 1452-1462.

Montagner, C. C., Jardim, W. F., Von Der Ohe, P. C., \& Umbuzeiro, G. A. (2014). Occurrence and potential risk of triclosan in freshwaters of São Paulo, Brazil: the need for regulatory actions. Environmental Science and Pollution Research International, 21, 1850-1858.

Montagner, C. C., Sodré, F. F., Acayaba, R. D., \& Vidal, C., Campestrini, I., Locatelli, M. A., Pescara, I. C. Albulquerque, A. F., Umbuzeiro, G. A., \& Jardim, W. F. (2019). Ten years-snapshot of the occurrence of emerging contaminants in drinking, surface and ground waters and wastewaters from São Paulo State, Brazil. Journal of the Brazilian Chemical Society, 30(3), 614-632.

Montagner, C. C., Vidal, C., \& Acayaba, R. D. (2017). Contaminantes emergentes em matrizes aquáticas do brasil: cenário atual e aspectos analíticos, ecotoxicológicos e regulatórios. Quimica Nova, 40(9), 1094-1110.

Moreira, J. C., Peres, F., Simões, A. C., Pignati, W. A., Dores, E. C., Vieira, S. N., Strüssmann, C., \& Mott, T. (2012). Contaminação de águas superficiais e de chuva por agrotóxicos em uma região do estado do Mato Grosso. Ciencia \& Saude Coletiva, 17, 557-1568. 
Moura, E. R. R. (2013). Determinação de Pesticidas no Rio Piquiri LC/MS/MS (Dissertação de mestrado). Universidade Tecnológica Federal do Paraná, Curitiba.

Padilha, C. F., \& Leitzke, F. L. S. (2013). Determination of female sex hormones in the Alto Iguaçu River Basin in the region of Curitiba- $\operatorname{Pr}$ (Trabalho de Conclusão de Curso). Departamento Acadêmico de Química e Biologia, Universidade Tecnológica Federal do Paraná, Curitiba.

Pedrouzo, M., Reverté, S., Borrull, F., Pocurull, E., \& Marcé, R. M. (2007). Pharmaceutical determination in surface and wastewaters using highperformance liquid chromatography-(electrospray)-mass spectrometry. Journal of Separation Science, 30(3), 297-303.

Peng, X., Yu, Y., Tang, C., Tan, J., Huang, Q., \& Wang, Z. (2008). Occurrence of steroid estrogens, endocrinedisrupting phenols, and acid pharmaceutical residues in urban riverine water of the Pearl River Delta, South China. The Science of the Total Environment, 397(1-3), 158-166.

Pessoa, G. P., De Souza, N. C., Vidal, C. B., Alves, J. A C., Firmino, P. I. M., Nascimento, R. F., \& Dos Santos, A. B. (2014). Occurrence and removal of estrogens in Brazilian wastewater treatment plants. The Science of the Total Environment, 490, 288-295.

Petrie, B., Barden, R., \& Kasprzyk-Hordern, B. (2014). A review on emerging contaminants in wastewaters and the environment: current knowledge, understudied areas and recommendations for future monitoring. Water Research, 72, 3-27.

Petrovic, M., Gros, M., \& Barcelo, D. (2006). Multi-residue analysis of pharmaceuticals in wastewater by ultraperformance liquid chromatographyquadrupole- time-of-flight mass spectrometry. Journal of Chromatography. A, 1124(1-2), 68-81.

Pivetta, G. G., \& Gastaldini, M. C. C. (2019). Presence of emerging contaminants in urban water bodies in southern Brazil. Journal of Water and Health, 17(2).

Quaresma, A. V. (2014). Monitoramento de microcontaminantes orgânicos por métodos cromatográficos acoplados à espectrometria de massa e elementos inorgânicos por fluorescência de raios - X por reflexão total nas águas da bacia do Rio Doce (Dissertação de mestrado). Universidade Federal de Ouro Preto, Ouro Preto.

Queiroz, F. B., Brandt, E. M. F., Aquini, S. F., Chernicharo, C. A. L., \& Afonso, R. J. C. F. (2012). Occurrence of pharmaceuticals and endocrine disruptores in raw sewage and their behavior in UASB reactors operated at different hydraulic retention times. Water Science and Technology, 66(12)

Queiroz, F. B., Silva, J. C., Aquino, S. F., Coutrim, M. X., \& Afonso, R. J. C. F. (2014). Determination of Endocrine Disrupters and Pharmaceuticals in Sewage Samples by Tandem Solid Phase Clean up/Extraction and High Performance Liquid Chromatography-Negative and Positive Electrospray High-Resolution Mass Spectrometry. Journal of the Brazilian Chemical Society, 25(2), 298-312.

Raimundo, C. C. M. (2011). Contaminantes emergentes em Água Tratada e seus Mananciais: Sazonalidade, Remoção e Atividade Estrogênica (Tese de doutorado). Departamento de Química Analítica, Instituto de Química, Universidade Estadual de Campinas, Campinas.

Rattier, M., \& Reungoat, J., Keller, J., \& Gernjak, W. (2014). Removal of micropollutants during tertiary wastewater treatment by biofiltration: role of nitrifiers and removal mechanisms. Water Research, 54, 8999.

Regnery, J., \& Puttmann, W. (2010). Occurrence and fate of organophosphorus flame retardants and plasticizers in urban and remote surface waters in Germany. Water Research, 44(14), 4097-4104.

Ribeiro, C., Pardal, M. A., Martinho, F., Margalho, R., Tritan, M. E., Rocha, E., \& Rocha, M. J. (2009). Distribution of endocrine disruptores in the Mondego River estuary, Portugal. Environmental Monitoring and Assessment, $149,183$.

Richardson, S. D., \& Kimura, S. (2016). Water analysis: emerging contaminants and current issues. Analytical Chemistry, 88, 546-582.

Richardson, S. D., \& Ternes, T. A. (2011). Water analysis: emerging contaminants and current issues. Analytical Chemistry, 83(12), 4614-4648.

Rodrigues, K. L. T., Sanson, A. L., Quaresma, A. D. V., Gomes, R. D. P., Da Silva, G. A., \& Afonso, R. J. D. C. F. (2014). Chemometric approach to optimize the operational parameters of ESI for the determination of contaminants of emerging concern in aqueous matrices by LC-IT-TOF-HRMS. Microchemical Journal, 117, 242-249.

Sanson, A. L. (2012). Estudo da extração e desenvolvimento de metodologia para determinação simultânea de microcontaminantes orgânicos em água superficial por GC-MS e métodos quimiométricos (Dissertação de mestrado). Universidade Federal de Ouro Preto, Ouro Preto. 
Santos, E. A. (2013). Contaminação por herbicidas em corpos hídricos da microbacia do Córrego Rico (SP) $e$ aspectos toxicológicos de atrazine a juvenis de Piaractus mesopotamicus (Tese de doutorado). Universidade Estadual Paulista, Jaboticabal.

Santos, L. H. M. L. M., Gros, M., Rodriguez-Mozaz, S., Delerue-Matos, C., Pena, A., Barceló, D., \& Montenegro, M. C. B. S. M. (2013). Contribution of hospital effluents to the load of pharmaceuticals in urban wastewaters: identification of ecologically relevant pharmaceuticals. The Science of the Total Environment, 461-462, 302316.

Sari, S., Ozdemir, G., Yangin-Gomec, C., Zengin, G. E., Topuz, E., Aydin, E., Pehlivanoglu-Mantas, E., \& Okutman Tas, D. (2014). Seasonal variation of diclofenac concentration and its relation with wastewater characteristics at two municipal wastewater treatment plants in Turkey. Journal of Hazardous Materials, 272, 155-164.

Simazaki, D., Kubota, R., Suzuki, T., Akiba, M., Nishimura, T., \& Kunikane, S. (2015). Occurrence of selected pharmaceuticals at drinking water purification plants in Japan and implications for human health. Water Research, 76, 187-200.

Sistema Nacional de Informações sobre Saneamento - SNIS. (2020). Diagnóstico dos Serviços de Água e Esgoto 2010-2018. Ministério do Desenvolvimento Regional. Recuperado em 3 de abril de 2020, de http://www.snis.gov.br/diagnosticos/agua-e-esgotos

Sodré, F. F. (2012). Interferentes Endócrinos como Contaminantes de preocupação emergentes: uma questão de saúde pública. Artigos Temáticos do AQQUA, 1, 1-8.

Sousa, D. N. R., Mozeto, A. A., Carneiro, R. L., \& Fadini, P. S. (2018). Spatio-temporal evaluation of emerging contaminants and their partitioning along a Brazilian watershed. Environmental Science and Pollution Research International, 25(5), 4607-4620.

Souza, R. R., Martins, E. A. J., Otomo, J. I., Furusawa, H. A., \& Pires, M. A. F. (2012). Determinação de plastificantes em água potável utilizando cromatografia gasosa e espectrometria de massas. Quimica Nova, 35(7), 1453-1458.

Sposito, J. C. V., Montagner, C. C., Casado, M., \& Navarro-Martín, L., Jut Solórzano, J. C., Piña, B., \& Grisolia, A. B. (2018). Emerging contaminants in Brazilian rivers: occurrence and effects on gene expression in zebrafish (Danio rerio) embryos. Chemosphere, 209, 696-704. http://dx.doi.org/10.1016/j.chemosphere.2018.06.046

Stasinakis, A. S., Thomaidis, N. S., Arvaniti, O. S., Asimakopoulos, A. G., Samaras, V. G., Ajibola, A., Mamais, D., \& Lekkas, T. D. (2013). Contribution of primary and secondary treatment on the removal of benzothiazoles, benzotriazoles, endocrine disruptors, pharmaceuticals and per fluorinated compounds in a sewage treatment plant. The Science of the Total Environment, 463-464, 1067-1075.

Stumpf, M., Ternes, T. A., Wilken, R., Rodrigues, S. V., \& Baumann, W. (1999). Polar drug residues in sewage and natural waters in the state of Rio de Janeiro, Brazil. The Science of the Total Environment, 225, 135-141.

Thomas, K. V., Silva, F. M. A., Lagford, K. H., Souza, A. D. L., Nizzeto, L., \& Waichman, A. V. (2014). Screening for selected human pharmaceuticals and cocaine in the urban streams of Manaus, Amazonas, Brazil. Journal of the American Water Resources Association, 50(2), 302-308.

Vulliet, E., \& Cren-Olivé, C. (2011). Screening of pharmaceuticals and hormones at the regional scale, in surface and groundwaters intended to human consumption. Environmental Pollution, 159(10), 2929-2934.

Xu, N., Xu, Y., Xu, S., Li, J., \& Tao, H. C. (2012). Removal of estrogens in municipal wastewater treatment plants: A Chinese perspective. Environmental Pollution, 165, 215-224.

Zorita, S., Mårtensson, L., \& Mathiasson, L. (2009). Occurrence and removal of pharmaceuticals in a municipal sewage treatment system in the south of Sweden. The Science of the Total Environment, 407(8), 2760-2770.

\section{Contribuições dos autores:}

Cindy Deina Farto: busca e seleção de artigos, planejamento e preparação e revisão do manuscrito.

Gilson Barbosa Athayde Júnior: planejamento e preparação e revisão do manuscrito.

Rennio Félix de Sena: revisão do manuscrito.

Raul Rosenhaim: revisão do manuscrito. 